\title{
A FURTHER NOTE ON DUAL TRIGONOMETRICAL SERIES
}

\author{
by C. J. TRANTER
}

(Received 7 January, 1960)

1. Introduction. This note discusses the determination of the coefficients $a_{n}$ in the dual trigonometrical series

$$
\left.\begin{array}{ll}
\sum_{n=1}^{\infty} n^{p} a_{n} \sin n x=F(x) & (0<x<c), \\
\sum_{n=1}^{\infty} a_{n} \sin n x=G(x) & (c<x<\pi),
\end{array}\right\}
$$

where $p= \pm 1$ and $F(x), G(x)$ are prescribed functions of $x$. It is shown that this problem and the corresponding one in which the sines in equations (1) are replaced by cosines are easily reduced to a form in which the results I have recently given in this journal [1] may be applied.

As with my previous paper on this subject, the analysis is purely formal and no attempt is made to give precise conditions for which the solution is valid.

2. The reduction of equations (1). Let $c_{n}$ be the coefficient of $\sin n x$ in the sine series representing $G(x)$ in the interval $0<x<\pi$ so that $\sum_{n=1}^{\infty} c_{n} \sin n x=G(x)$ and

$$
c_{n}=\frac{2}{\pi} \int_{0}^{\pi} G(x) \sin n x d x
$$

If

$$
A_{n}=a_{n}-c_{n}
$$

equations (1) can therefore be written

$$
\left.\begin{array}{ll}
\sum_{n=1}^{\infty} n^{y} A_{n} \sin n x=f(x) & (0<x<c) \\
\sum_{n=1}^{\infty} A_{n} \sin n x=0 & (c<x<\pi)
\end{array}\right\}
$$

where

$$
f(x)=F(x)-\sum_{n=1}^{\infty} n^{p} c_{n} \sin n x .
$$

The problem of determining the coefficients $A_{n}$ from equations (4) is precisely that given in $\S \S 2,5$ of my previous paper [1] and the problem is therefore formally solved.

3. An example. In equations (1) take $p=1$,

$$
F(x)=\sin x /(\cos c-\cos x) \text { and } G(x)=\pi-x .
$$

From equations (2) and (5),

$$
c_{n}=\frac{2}{\pi} \int_{0}^{\pi}(\pi-x) \sin n x d x=\frac{2}{n},
$$


and

$$
\begin{aligned}
f(x) & =\frac{\sin x}{\cos c-\cos x}-2 \sum_{n=1}^{\infty} \sin n x \\
& =\frac{\sin \frac{1}{2} x \cos \frac{1}{2} x}{\sin ^{2} \frac{1}{2} x-\sin ^{2} \frac{1}{2} c}-\cot \frac{1}{2} x,
\end{aligned}
$$

if we take the Cesàro sum of the sine series. Writing $\sin \frac{1}{2} x=\rho \sin \frac{1}{2} c$, we obtain

$$
f\left\{2 \sin ^{-1}\left(\rho \sin \frac{1}{2} c\right)\right\}=\frac{\left(1-\rho^{2} \sin ^{2} \frac{1}{2} c\right)^{t}}{\rho\left(\rho^{2}-1\right) \sin \frac{1}{2} c} .
$$

Substituting this in equation (17) of my previous paper [1], we get

$$
\chi(s)=\frac{4}{\pi s \sin \frac{1}{2} c} \int_{0}^{s} \frac{d \rho}{\left(\rho^{2}-1\right)\left(s^{2}-\rho^{2}\right)^{3}} .
$$

By writing $\rho=s \sin \theta$, the definite integral is easily evaluated and we find

$$
\chi(s)=-\frac{2}{s\left(1-s^{2}\right)^{\frac{1}{2}} \sin \frac{1}{2} c} .
$$

Substitution in equation ( 7 ) of my previous paper then gives

$$
A_{n}=-4 n \sin ^{2} \frac{1}{2} c \int_{0}^{1} s\left(1-s^{2}\right)^{-\frac{1}{3}} F\left(1+n, 1-n ; 2 ; s \sin ^{2} \frac{1}{2} c\right) d s .
$$

The definite integral in (8) can be evaluated as follows. From Watson [2, p. 401], since $0<s \sin \frac{1}{2} c<1$,

$$
F\left(1+n, 1-n ; 2 ; s^{2} \sin ^{2} \frac{1}{2} c\right)=\frac{1}{n s \sin \frac{1}{2} c} \int_{0}^{\infty} J_{2 n}(t) J_{1}\left(t s \sin ^{2} \frac{1}{2} c\right) d t,
$$

and substitution in (8) gives, after interchanging the order of integration,

$$
\begin{aligned}
A_{n} & =-4 \sin \frac{1}{2} c \int_{0}^{\infty} J_{2 n}(t) d t \int_{0}^{1}\left(1-s^{2}\right)^{-1} J_{1}\left(t s \sin \frac{1}{2} c\right) d s \\
& =-4 \sin \frac{1}{2} c \int_{0}^{\infty} J_{2 n}(t) d t \int_{0}^{\pi / 2} J_{1}\left(t \sin \frac{1}{2} c \sin \alpha\right) d \alpha \quad(s=\sin \alpha), \\
& =-4 \int_{0}^{\infty}\left\{1-\cos \left(t \sin \frac{1}{2} c\right)\right\} J_{2 n}(t) \frac{d t}{t}
\end{aligned}
$$

when use is made of Watson [2, pp. 374, 333]. The value of this integral is again given by Watson [2, p. 405] and we find that

$$
A_{n}=\frac{2}{n}(\cos n c-1)
$$

Using (3) and (6), we find that the required value of the coefficient $a_{n}$ is given by

$$
a_{n}=A_{n}+c_{n}=A_{n}+\frac{2}{n}=\frac{2}{n} \cos n c .
$$


4. The similar problem for cosine series. Here we wish to determine the coefficients $b_{n}$ in the dual series

$$
\left.\begin{array}{ll}
\sum_{n=1}^{\infty} n^{p} b_{n} \cos n x=P(x) & (0<x<c), \\
\sum_{n=1}^{\infty} b_{n} \cos n x=Q(x) & (c<x<\pi),
\end{array}\right\}
$$

where $p= \pm 1$ and $P(x), Q(x)$ are now prescribed. Writing

$$
a_{n}=b_{n} / n, \quad F(x)=\int_{0}^{x} P(t) d t, \quad G(x)=-\int_{x}^{\pi} Q(t) d t
$$

we easily see, by integrating with respect to $x$ between $0, x$ and $x, \pi$ respectively, that equations (9) reduce to equations (1) and this problem can be solved as before.

\section{REFERENCES}

1. C. J. Tranter, Dual trigonometrical series, Proc. Glasgow Math. Assoc. 4 (1959), 49-57.

2. G. N. Watson, Theory of Bessel functions (Cambridge, 1944).

Royal Muitary College of Science

SHRIVENHAM

\section{SOME TRIPLE INTEGRAL EQUATIONS}

by C. J. TRANTER

(Received 3 March, 1960)

1. Introduction. Potential problems in which different conditions hold over $t w o$ different parts of the same boundary can often be conveniently reduced to the solution of a pair of dual integral equations. In some problems, however, the boundary condition is such that different conditions hold over three different parts of the boundary and, in such cases, the integral equations involved are frequently of the form

$$
\left.\begin{array}{ll}
\int_{0}^{\infty} \phi(u) J_{\nu}(r u) d u=f(r) & (0<r<a), \\
\int_{0}^{\infty} u^{2 p} \phi(u) J_{v}(r u) d u=g(r) & (a<r<b), \\
\int_{0}^{\infty} \phi(u) J_{v}(r u) d u=0 & (b<r<\infty),
\end{array}\right\}
$$

